\title{
Ovarian Function Preservation in Patients With Cervical Cancer Undergoing Hysterectomy and Ovarian Transposition Before Pelvic Radiotherapy
}

Technology in Cancer Research \& Treatment

Volume 20: 1-9

(C) The Author(s) 2021

Article reuse guidelines:

sagepub.com/journals-permissions DOI: I0.1 I77/I53303382। I042। 40 journals.sagepub.com/home/tct

@SAGE

\author{
Wonguen Jung, MD, PhD', Yun H. Kim, MD, PhD', \\ and Kyung S. Kim, MD, PhD' ${ }^{\prime}$
}

\begin{abstract}
To examine the factors associated with ovarian failure (OF) and assess the effectiveness of ovarian transposition (OT) before pelvic irradiation for preserving ovarian function in patients with cervical cancer (CC) undergoing hysterectomy. During 2003 to 2017, patients who underwent hysterectomy with preservation of one or both ovaries were retrospectively enrolled. Patients were divided into 4 groups, depending on whether radiotherapy (RT) and OT were performed: group I, RT(+) and OT(+); group 2, RT(+) and OT(-); group 3, RT(-) and OT(+); group 4, RT(-) and OT(-). OF was defined as serum folliclestimulating hormone levels of $\geq 30 \mathrm{mlU} / \mathrm{mL}$. Sixty-six patients $(59$ [89.4\%] invasive CC and 7 [ $10.6 \%]$ cervical intraepithelial neoplasia) were included. The 2-year OF-free survival rate was $61.4 \%$ (95\% confidence interval [Cl] 37.8-86.0), 0\%, 91.7\% (95\% Cl 76.0-100), and 75.8\% (95\% Cl 58.2-93.4) for groups I, 2, 3, and 4, respectively. In groups I and 2 receiving RT, OT, and combination of external beam radiotherapy and vaginal brachytherapy were associated with OF on multivariate analysis (MVA) ( $P$-value $=.002$ and .046, respectively). In groups 3 and 4 without RT, older age (40 years old) and OT did not affect OF; however, the number of remaining ovaries was independently associated with OF in MVA $(P=.035)$. OT could effectively preserve ovarian function in patients treated with adjuvant RT, while OT procedure itself did not affect ovarian failure. OT should be considered in the management of premenopausal cervical cancer patients.
\end{abstract}

\section{Keywords}

ovarian transposition, ovarian function, ovarian preservation, cervical cancer, radiotherapy

\begin{abstract}
Abbreviations
CCRT, concurrent chemoradiotherapy; EBRT, external beam radiation therapy; FSH, follicle-stimulating hormone; FIGO, International Federation of Gynecology and Obstetrics; MVA, multivariate analysis; OFFS, ovarian failure-free survival; OT, ovarian transposition; RT, radiation therapy; VB, vaginal brachytherapy
\end{abstract}

Received: February 24, 2021; Revised: July 13, 2021; Accepted: August 9, 2021.

\section{Introduction}

The incidence rates of cervical cancer have been decreasing in Korea and it is the third most common cancer in women of reproductive age (15-34 years). ${ }^{1}$ Patient with early-stage cervical cancer who undergo radical hysterectomy and pelvic lymphadenectomy are treated with adjuvant radiation therapy (RT) or concurrent chemoradiotherapy (CCRT) according to pathological risk factors.

\footnotetext{
'Ewha Womans University College of Medicine, Seoul, Republic of Korea

Corresponding Author:

Kyung S. Kim, Department of Radiation Oncology, Ewha Womans University College of Medicine, 260, Gonghang-daero, Gangseo-gu, Seoul, 07804, Republic of Korea.

Email: happyend67@gmail.com
} 
However, when ovaries are within the field of pelvic irradiation, ovarian failure can occur after adjuvant pelvic RT. ${ }^{2-4}$ Because oocytes are sensitive to radiation damage, ionizing radiation can cause irreversible damage such as oocyte loss and decline in follicle number, leading subsequently to irregular menstruation and ovarian failure. ${ }^{5-7}$

Ovarian failure for premenopausal women is also associated with cardiovascular disease and osteoporosis in addition to poor quality of life as a result of hot flashes and vaginal dryness. ${ }^{8}$ Thus, it is important to improve the quality of life and maintain fertility by preserving ovarian function in premenopausal women with cervical cancer receiving RT.

Ovarian transposition (OT) before pelvic irradiation is used to minimize ovarian follicle exposure to radiation and to preserve ovarian function by transposing the ovaries outside the irradiation field. $^{9-11}$

The purpose of this study was to examine the factors associated with ovarian failure and to evaluate the effectiveness of OT before pelvic irradiation for preserving ovarian function in patients with cervical cancer who underwent a hysterectomy.

\section{Methods}

\section{Patients}

Patients who underwent a hysterectomy in our institution from April 2003 to March 2017 were retrospectively reviewed. The present study was approved by the institutional review board of our institution (approval number: 2019-11-005 at Ewha

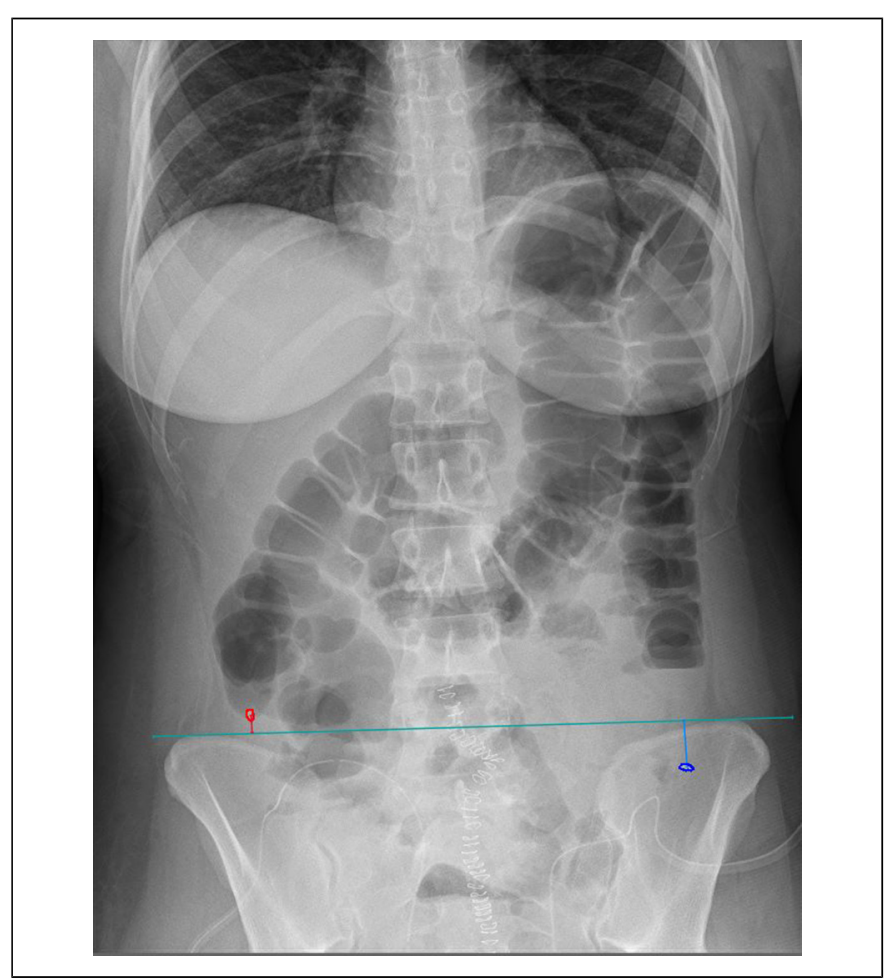

Figure 1. Erect abdominal X-ray of a patient who received bilateral ovarian transposition (right ovary: red, left ovary: blue).
Womans University Seoul Hospital). The study inclusion criteria were as follows: (1) patients with histologically confirmed uterine cervical cancer who were $>18$ years old and $<50$ years old; (2) patients with preservation of one or both ovaries after hysterectomy; and (3) patients with serum follicle-stimulating hormone (FSH) level measured in peripheral blood during follow-up. OT during radical hysterectomy was performed in younger patients or those who wanted to preserve ovarian function and were candidates for adjuvant RT or CCRT.

Patients were divided into 4 groups, depending on whether the patients received OT during hysterectomy and adjuvant RT. Patients in group 1 received both OT and RT. Patients in group 2 received adjuvant RT but not OT. Patients in group 3 received OT but not RT. Patients in group 4 did not undergo both OT and RT.

\section{Postoperative Treatment}

A total adjuvant external beam radiotherapy (EBRT) dose of 41.4 to 54.0 Gy was delivered to the whole pelvis. Vaginal brachytherapy (VB) was administered with an Ir-192 brachytherapy unit (Microselectron; Nucletron), after EBRT. Additional VB dose of 15 to $24 \mathrm{~Gy}$ in 4 to 6 fractions was prescribed at $0.5 \mathrm{~cm}$ depth from the surface of the cylinder.

\section{Evaluation of Location of the Transposed Ovary}

The transposed ovary was identified by the placement of the radio-opaque surgical clips at the time of hysterectomy as shown by the abdominal X-ray. For patients without abdominal $\mathrm{X}$-rays, scout views of abdomen pelvis computed tomography were used. The distance of the transposed ovary was calculated as the perpendicular length between the surgical clip of the transposed ovary and the horizontal line to the iliac crest (Figure 1). When bilateral OT was performed, the location of the transposed ovary was measured by the distance from the higher transposed ovaries.

\section{Evaluation of Ovarian Failure}

The ovarian function in the present study was assessed by measurement of the patient's serum FSH levels after surgery as in previous studies. ${ }^{10,12}$ In patients aged under 45 years old during the follow-up period, the lowest serum FSH level data was collected. In patients aged over 45 years old during the follow-up period, the latest serum FSH level data without ovarian failure was collected and considered censored. Ovarian failure was defined as serum FSH levels of $30 \mathrm{mIU} /$ $\mathrm{mL}$ or higher. Ovarian failure-free survival (OFFS) was defined as the time from the end date of RT in groups 1 and 2 or the surgery date in groups 3 and 4 to the date of ovarian failure. 


\section{Statistical Analyses}

Survival curves were generated using the Kaplan-Meier method, and a log-rank test was used to compare survival between groups. Cox's regression model was used to analyze independent risk factors for ovarian failure. $P$-values $<.05$ were considered statistically significant. Statistical analyses were performed using SPSS software version 18.0 (SPSS Inc).

\section{Results}

A total of 66 consecutive cervical cancer patients were enrolled in this study. The patients and tumor characteristics are summarized in Table 1 . The median age at hysterectomy was 39.5 (26.7-48.4) years. OT was performed on a single ovary in 20 patients and on both ovaries in 9 patients. Twenty-three patients received adjuvant EBRT with a median dose of 50.4 Gy (range: 41.4-54) in groups 1 and 2. In group 1, 1 and 15 patients were treated with intensity-modulated radiotherapy (IMRT) and three-dimensional conformal radiotherapy (3D-CRT). In group 2, 1 and 6 patients received twodimensional RT and 3D-CRT. The ovarian maximal dose was confirmed in 17 transposed ovaries of 10 patients, and the median dose was $557.8 \mathrm{cGy}$ (range: 140.2-2418.3). Five of these patients received an additional high-dose-rate VB with a median dose of 24 Gy (range: 15-24). Adjuvant chemotherapy was performed in $25.7 \%$ ( 17 of 66 ) of the patients. Of these, 15 patients in groups 1 and 2 received concurrent chemoradiation. Thirteen of 29 patients (44.8\%) who underwent OT developed a benign ovarian cyst, and the median duration between surgery or RT and the date of development of ovarian cysts was 24.9 months (range: 0.9-142.6).

The median follow-up of patients from surgery or end of RT until the serum FSH level date of ovarian function was 15.4 months (range: $0.2-126.3$ ). About $63.6 \%$ (42 of 66) of the patients had a normal ovarian function. The rates of preservation of ovarian function by group are as follows: $56.3 \%$ ( 9 of 16 ) in group 1, $0 \%$ (0 of 7) in group 2, 84.6\% (11 of 13) in group 3 , and $73.3 \%$ (22 of 30) in group $4(P=.001)$. OFFS at 2 years was $61.4 \%$ for group $1,0 \%$ for group $2,91.7 \%$ for group 3 , and $75.8 \%$ for group $4(P<.001$, Figure 2$)$.

In group 1 , age $(P=.014)$, VB $(P=.003)$, and the location of transposed ovaries $(P=.007)$ were associated with OFFS in univariate analysis but not in MVA. All 4 patients treated with EBRT plus VB had transposed ovary below the iliac crest (median $-1.9 \mathrm{~cm}$, range $-5.1-[-0.5]$ ), and all of them experienced early ovarian failure (median OFFS 2.3 months, 95\% CI 0-6.8). The number of transposed ovaries was not significantly associated with ovarian failure in this group.

For patients in groups 1 and 2 who received RT, associations between prognostic factors and OFFS are shown in Table 2. OT was only significantly associated with OFFS in univariate analysis $(P=.001)$. MVA demonstrated that ovaries without transposition and VB boost were significant prognostic factors affecting OFFS. The hazard ratios were $3.452(95 \% \mathrm{CI}, 1.023-11.654 ; P=.002)$, and 6.362
(95\% CI, 1.932-0.963, $P=.046)$ for ovaries without transposition and VB boost, respectively. Additional VB showed significant difference in OFFS versus patients who received EBRT alone: 2 years OFFS $0 \%$ versus $83.3 \%(P$ $=.03$, Figure 3 ).

In patients in groups 3 and 4 who did not receive RT, the number of residual ovaries was the only significant prognostic factor affecting ovarian failure in univariate analysis and MVA ( $P=.035$, Table 3 , Figure 4$)$. Older age ( $\geq 40$ years) and OT did not affect ovarian failure.

\section{Discussion}

The current study showed that OT could effectively preserve ovarian function in patients treated with adjuvant RT, and ovarian failure clearly occurs when ovaries are located in the radiation field. Moreover, the preservation of both ovaries resulted in a significant increase in OFFS in the group without RT.

OT is a surgery to transpose the ovaries out of the field of radiation to protect them from radiation damage. ${ }^{13}$ Hoekman et al ${ }^{11}$ noted that the 5 -year ovarian survival rate was $60.3 \%$ in patients who underwent OT before RT, whereas all patients who received RT without OT had an ovarian failure, which is similar to our study. The degree of ovarian damage and ovarian failure is affected by the radiation dose of ovaries. ${ }^{14,15}$ Several studies reported that the OT before pelvic RT reduces the ovarian dose received to approximately $5 \%$ to $10 \%$ of that in the untransposed ovaries. ${ }^{6,7,16}$ Winarto et $\mathrm{al}^{7}$ demonstrated that the lateral transposed ovaries receive about 0.45 to $4.5 \mathrm{~Gy}$, corresponding to $1 \%$ to $10 \%$ of the total RT dose of $45 \mathrm{~Gy}$. Our study showed that the rate of OFFS at 2 years was $61.4 \%$ for patients with OT before pelvic RT, whereas all patients who received adjuvant RT without OT had an ovarian failure. Therefore, OT before pelvic RT could be helpful for preserving ovarian function for candidates for adjuvant RT or CCRT. Du and $\mathrm{Qu}^{17}$ evaluated the association between ovarian limited dose and ovarian function and suggested an ovarian radiation dose in cervical cancer patients who underwent OT and were treated with IMRT. This study showed that ovarian radiation dose to $\mathrm{V}_{7.5}<26 \%$ can prevent ovarian failure in IMRT.

Our results showed that in groups 1 and 2,78\% (18 of 23) of the patients received EBRT alone, and 22\% (5 of 23) had an additional VB in addition to EBRT. The rates of ovarian preservation at 2 years after RT were $54.5 \%$ in EBRT alone and $0 \%$ in patients with EBRT plus VB. Although our study showed an association between VB and ovarian failure, other studies have not found such a correlation. Morice et $\mathrm{al}^{18}$ reported that the ovarian survival was $100 \%$ (11 of 11$)$ after OT, $90 \%$ (53 of 59) after OT and VB, and 60\% (15 of 25) after OT, VB, and EBRT. Clough et $\mathrm{al}^{19}$ reported that the mean dose of the transposed ovary was $2 \mathrm{~Gy}$, whereas the untransposed ovary was 32.2 Gy on pelvic dosimetry of patients who received 65 Gy with intrauterine and endovaginal brachytherapy alone. However, a recent metaanalysis by Laios et $\mathrm{al}^{20}$ also reported that ovarian function preservation was 


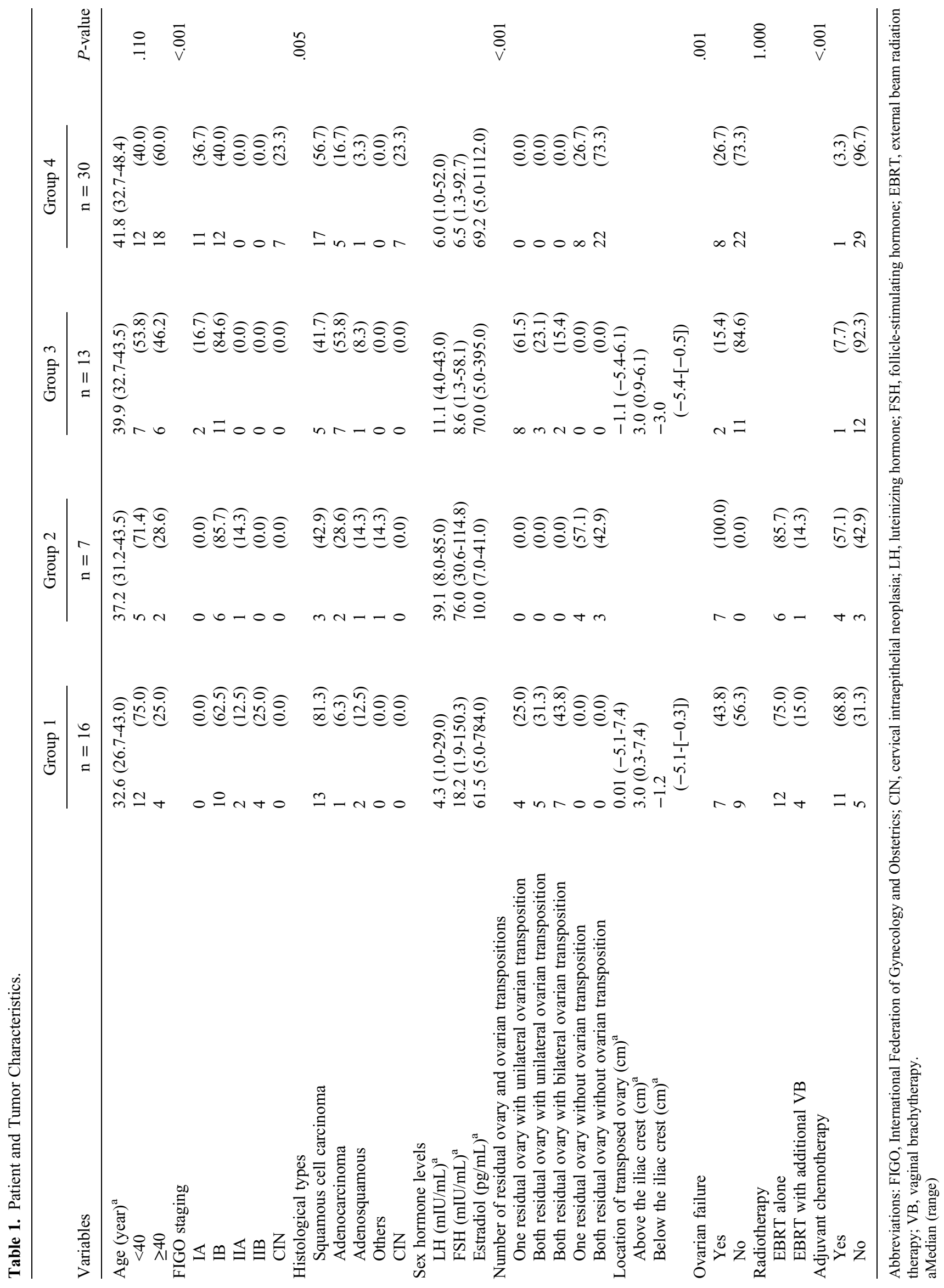




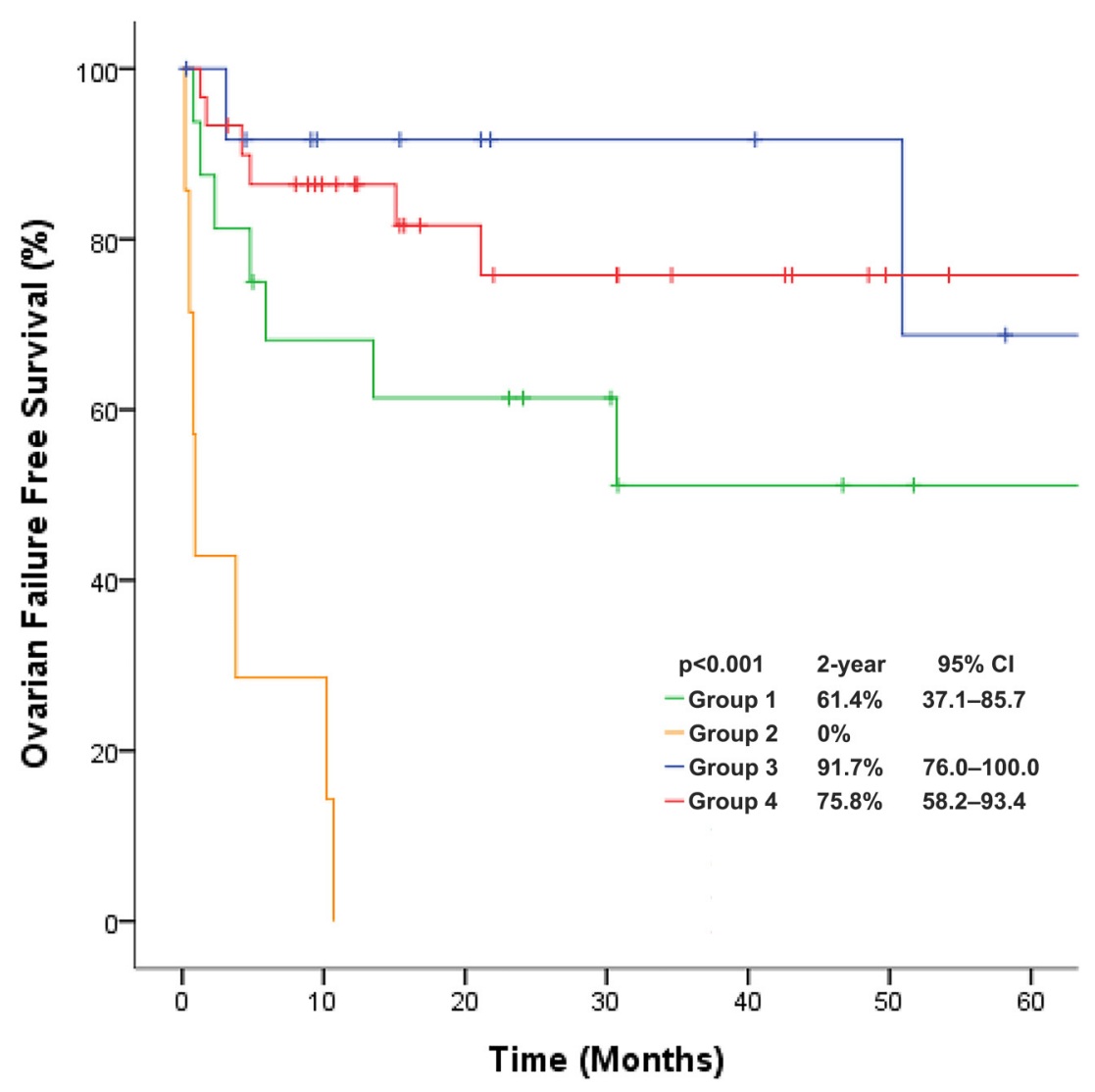

Figure 2. Ovarian failure-free survival according to the groups.

Table 2. Univariate and Multivariate Analysis for Ovarian Failure-Free Survival Outcomes in Groups 1 and 2.

\begin{tabular}{|c|c|c|c|c|c|c|}
\hline \multirow[b]{2}{*}{ Variable } & \multirow[b]{2}{*}{$\mathrm{n}$} & \multicolumn{2}{|c|}{ Univariate } & \multicolumn{3}{|c|}{ Multivariate } \\
\hline & & $\begin{array}{l}\text { 2-year } \\
\text { rate }\end{array}$ & $P$ & & $\mathrm{R}(95 \% \mathrm{CI})$ & $P$ \\
\hline Age & & & .056 & 2.035 & $(0.635-6.518)$ & .232 \\
\hline$<40$ years & 17 & $52.9 \%$ & & & & \\
\hline$\geq 40$ years & 6 & $0 \%$ & & & & \\
\hline Residual ovary & & & .871 & & & \\
\hline Both ovary & 15 & $45.0 \%$ & & & & \\
\hline Single ovary & 8 & $37.5 \%$ & & & & \\
\hline $\begin{array}{l}\text { Ovarian } \\
\text { transposition }\end{array}$ & & & .001 & 3.452 & $(1.023-11.654)$ & .002 \\
\hline Yes & 16 & $61.4 \%$ & & & & \\
\hline No & 7 & $0 \%$ & & & & \\
\hline Radiotherapy & & & .065 & 6.362 & $(1.931-20.963)$ & .046 \\
\hline EBRT alone & 18 & $54.5 \%$ & & & & \\
\hline $\begin{array}{l}\text { EBRT with } \\
\text { additional VB }\end{array}$ & 5 & $0 \%$ & & & & \\
\hline $\begin{array}{l}\text { Adjuvant } \\
\text { chemotherapy }\end{array}$ & & & .691 & & & \\
\hline No & 8 & $33.3 \%$ & & & & \\
\hline Yes & 15 & $46.7 \%$ & & & & \\
\hline
\end{tabular}

Abbreviations: OFFS, ovarian failure-free survival; HR, hazard ratio; CI, confidence interval; EBRT, external beam radiation therapy; VB, vaginal brachytherapy. similar in $91 \%$ of patients with OT and VB compared to $93 \%$ of patients with OT alone, whereas $62 \%$ of patients who underwent EBRT with or without VB after OT showed ovarian function preservation. This metaanalysis showed that patients with OT and VB alone have a good ovarian function as OT alone and the addition of EBRT increases the ovarian failure rates, and EBRT had a greater impact on ovarian failure than VB. The current study showed that in group 1, all 4 patients treated with EBRT plus VB had transposed ovary below the iliac crest (median $-1.9 \mathrm{~cm}$, range $-5.1-[-0.5]$ ), and all experienced early ovarian failure. Several studies reported that the location of the transposed ovary was associated with ovarian survival, and the distance between the edge of the RT field and the transposed ovaries affects the successful preservation of ovarian function. Winarto et $\mathrm{al}^{7}$ suggested the above iliac crest as the suboptimal placement of the ovary. Yoon et $\mathrm{al}^{10}$ also noted that the location of transposed ovaries may be associated with ovarian failure after RT. They suggested that young women with early-stage cervical cancer who might be a candidate for postoperative RT should be transposed to the ovaries as highly as possible during a radical hysterectomy to avoid ovarian failure. Hwang et $\mathrm{al}^{12}$ suggested that the location of transposed ovary more than $1.5 \mathrm{~cm}$ above the iliac crest was recommended to preserve ovarian function after pelvic RT in uterine cervical cancer. In addition, even if the ovaries are sufficiently outside the RT field, ovarian damage can also occur as 


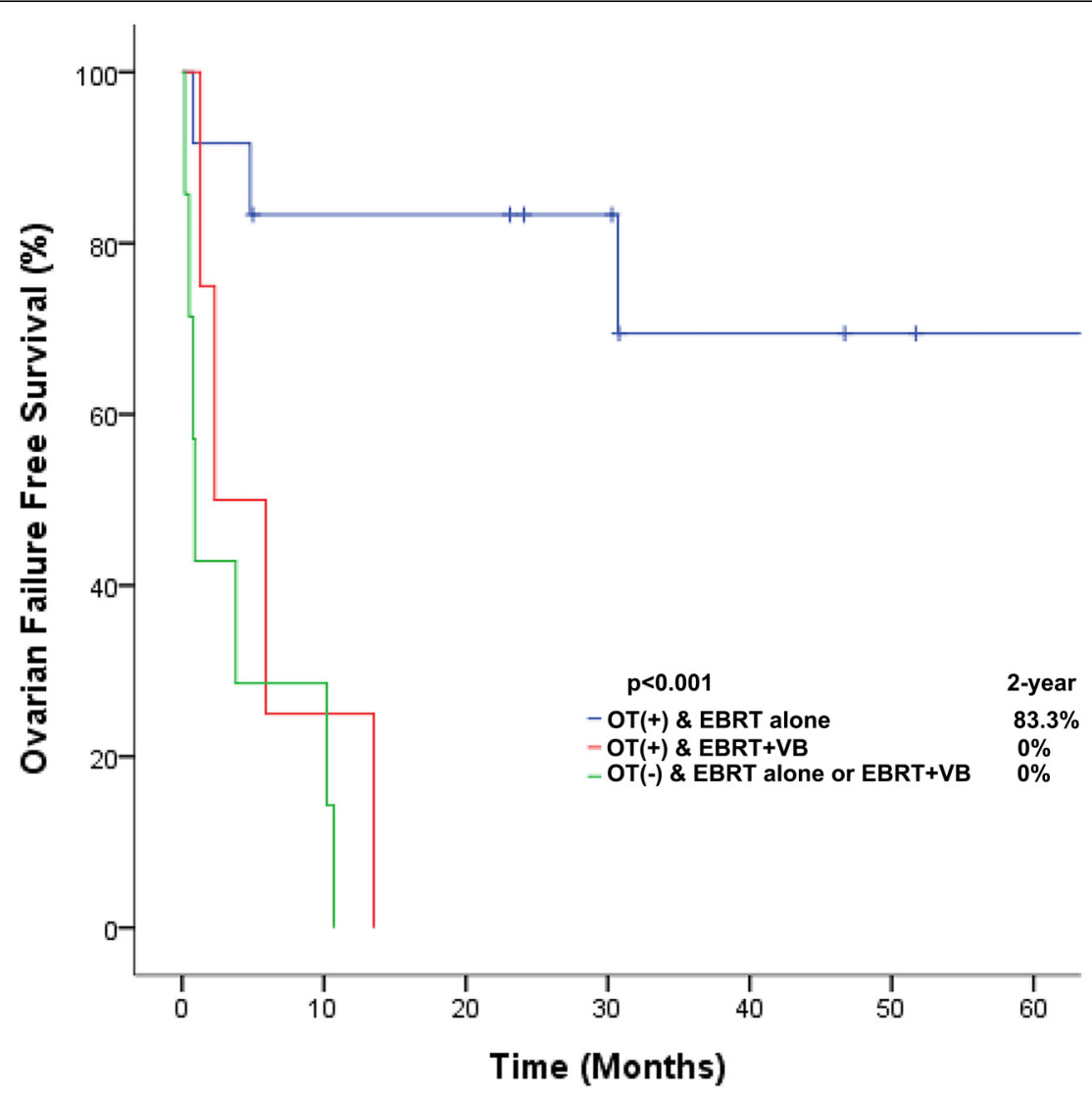

Figure 3. Ovarian failure-free survival after radiation therapy by external beam radiation therapy or vaginal brachytherapy. OT, ovarian transposition; EBRT, external beam radiation therapy; VB, vaginal brachytherapy.

a result of scattered radiation doses. Van Eijkeren et $\mathrm{al}^{21}$ reported that patients experienced ovarian failure if the scatter radiation dose to the transposed ovaries was more than

Table 3. Univariate and Multivariate Analysis for Ovarian Failure-Free Survival Outcomes in Groups 3 and 4.

\begin{tabular}{|c|c|c|c|c|c|c|}
\hline \multirow[b]{2}{*}{ Variable } & \multirow[b]{2}{*}{$\mathrm{n}$} & \multicolumn{2}{|c|}{ Univariate } & \multicolumn{3}{|c|}{ Multivariate } \\
\hline & & $\begin{array}{l}\text { 2-year } \\
\text { rate }\end{array}$ & $P$ & & $\mathrm{R}(95 \% \mathrm{CI})$ & $P$ \\
\hline Age & & & .182 & 3.723 & $(0.743-18.658)$ & .110 \\
\hline$<40$ years & 19 & $94.4 \%$ & & & & \\
\hline$\geq 40$ years & 24 & $67.7 \%$ & & & & \\
\hline Residual ovary & & & .047 & 4.633 & $(1.112-19.310)$ & .035 \\
\hline Both ovary & 27 & $84.6 \%$ & & & & \\
\hline Single ovary & 16 & $73.3 \%$ & & & & \\
\hline $\begin{array}{l}\text { Ovarian } \\
\text { transposition }\end{array}$ & & & .685 & & & \\
\hline Yes & 13 & $91.7 \%$ & & & & \\
\hline No & 30 & $75.8 \%$ & & & & \\
\hline $\begin{array}{l}\text { Adjuvant } \\
\text { chemotherapy }\end{array}$ & & & .361 & & & \\
\hline No & 41 & $81.9 \%$ & & & & \\
\hline Yes & 2 & $50.0 \%$ & & & & \\
\hline
\end{tabular}

Abbreviations: HR, hazard ratio; CI, confidence interval.
$300 \mathrm{cGy}$. The current study showed that in group 1, the location of the transposed ovary was associated with OFFS in univariate analysis. After all, the radiation dose to the ovary is the critical factor of $\mathrm{OF}$, the ovaries should be transposed as high and laterally as possible from the pelvic brim, and effort to reduce irradiated dose to the ovary using IMRT is essential to prevent OF.

The degree of ovarian damage is dependent on the patient's age as well as the irradiated ovarian dose and type of gonadotoxic agent used. ${ }^{6,15,22,23}$ OT has been generally suggested for patients aged under 40 years old because the patient's age is also known to be a crucial factor to determine the success of OT for candidate patients. ${ }^{4,15,18,19}$ Morice et al ${ }^{18}$ reported the limited value of OT in patients over 40 years because they have an intrinsically decreased fertilization possibility as well as a much higher risk for ovarian failure despite OT. They noted that the rate of menopause after hysterectomy is clearly too high to recommend OT to patients tagged 40 years and older treated for cervical cancer. It has been reported that for patients who undergo hysterectomy with OT procedure, ovarian failure occurred in $14.3 \%$ ( 1 of 7 ) of patients under 40 years of age, compared with $85.7 \%$ (6 of 70 ) in patients over 40 years of age. ${ }^{24}$ In contrast to previous studies, our study demonstrated that older age ( $\geq 40$ years old) did not affect ovarian failure in the groups with RT (groups 1 and 2) or without RT (groups 3 and 4). Moreover, the OT procedure 


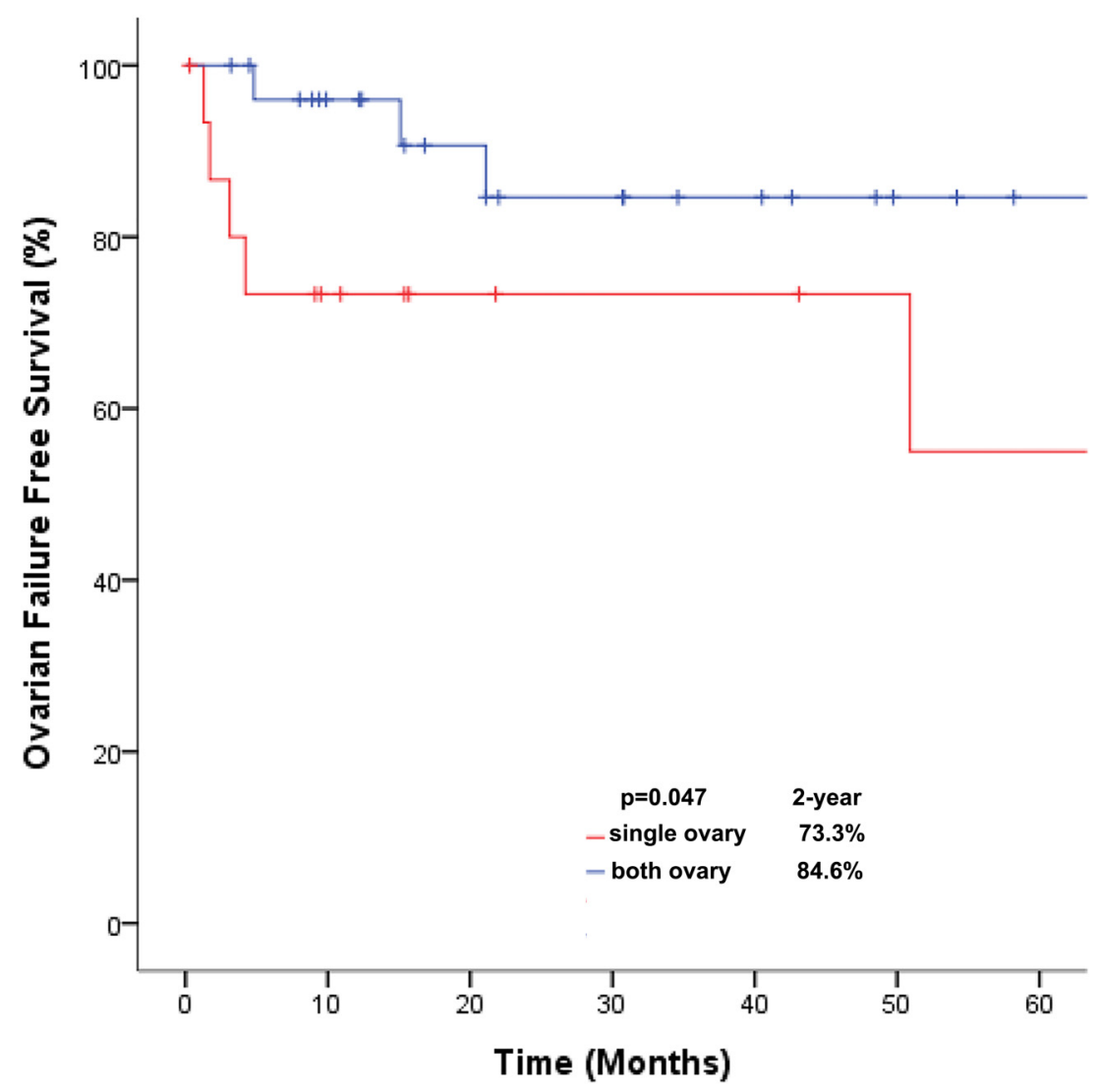

Figure 4. Ovarian failure-free survival by a number of residual ovaries.

itself did not affect ovarian failure in patients without RT. The median age at menopause among Korean women is approximately 50 years. $^{25}$ The age of menopause ranged from 33 to 61 years, with $88.2 \%$ between 45 and 55 years, $9.4 \%$ under 44 years, and $2.4 \%$ over 56 years. Considering that ovarian failure can affect the quality of life as well as lead to hot flashes, vaginal dryness, and cardiovascular disease, OT could be sufficiently considered even in Korean women aged over 40 years.

The sequelae of OT such as ovarian cysts and ovarian torsion are considered when performing OT. ${ }^{26}$ Morice et al ${ }^{18}$ reported that for patients undergoing radical hysterectomy and OT, the development of benign ovarian cyst was reported in 23\% (22 of 95) patients, 3 of whom required surgical intervention. The rates of ovarian cysts were $18 \%$ (2 of 11 ) in the radical hysterectomy alone group, 34\% (20 of 59) in the group that underwent radical hysterectomy and VB, and $0 \%$ (0 of 29) in patients who underwent a radical hysterectomy, EBRT and VB $(P=.01)$ in their study. Chambers et $\mathrm{al}^{27}$ reported that the incidence of symptomatic ovarian cysts was $7.4 \%$ in patients who underwent radical hysterectomy alone and $7 \%$ in patients who also received RT as compared to $24 \%$ in those who underwent OT, and most ovarian cysts required surgical intervention. The current study showed that OT did not affect ovarian failure in the group without RT. Although OT did not affect ovarian failure, consideration should be given to the surgical morbidity mentioned in previous studies.

The number of remaining ovaries can affect ovarian failure. In a previous study, Buekers et $\mathrm{al}^{3}$ noted that ovarian failure was significantly different between patients of unilateral oophorectomy and contralateral OT and those with ovaries preserved and transposed ( 4 months vs 43 months, $P=.003$ ). All patients with unilateral oophorectomy had ovarian failure by 1 year after treatment, but $41 \%$ of patients with both ovaries retained maintained ovarian function after 1 year in their study. Our result showed that in the group without RT, the number of remaining ovaries was an independent factor for ovarian failure, which is similar to the previous study. However, unilateral oophorectomy also showed a favorable outcome with $75.8 \%$ of 2 -year OFFS in patients without RT in the current study.

The current study had several limitations. First, this study was retrospective; therefore, serum FSH levels were not collected regularly during the follow-up period. Second, the number of patients included in this study was small. The number of patients in groups 1, 2, and 3 was less than 30, making it difficult to divide into subgroups for analysis. Third, this study did not address the dosimetry effects of the transposed ovary on ovarian failure. Since the ovarian dose is associated with ovarian function preservation, analysis on the dosimetric impact of pelvic irradiation received by the transposed ovary is required in the future. 


\section{Conclusions}

OT could effectively preserve ovarian function in patients treated with adjuvant RT. Moreover, the preservation of both ovaries resulted in a significant increase in OFFS in the group without RT. In addition, the OT procedure itself did not affect ovarian failure, while unilateral oophorectomy showed inferior ovarian survival than those who had both ovaries. These findings may help to inform preservation strategies of ovarian function in young patients with cervical cancer.

\section{Authors' Contributions}

Kyung Su Kim and Wonguen Jung conceived and designed the research. Wonguen Jung analyzed the data and wrote the manuscript with support from Kyung Su Kim and Yun Hwan Kim. Kyung Su Kim supervised the findings of this study. All authors discussed the results and commented on the manuscript.

\section{Declaration of Conflicting Interests}

The authors declared no potential conflicts of interest with respect to the research, authorship, and/or publication of this article.

\section{Ethics Approval and Consent to Participate}

This study was reviewed and approved by the institutional review board of Ewha Womans University Seoul Hospital (approval number: 2019-11-005). The participants' informed consent requirement was waived due to the retrospective nature of this study.

\section{Funding}

This research was supported by Basic Science Research Program through the National Research Foundation of Korea (NRF) funded by the Ministry of Education (2020R1I1A1A01072873).

\section{ORCID iD}

Kyung S. Kim (iD https://orcid.org/0000-0002-3480-7078

\section{References}

1. Jung K-W, Won Y-J, Kong H-J, Lee ES. Cancer statistics in Korea: incidence, mortality, survival, and prevalence in 2016. Cancer Res Treat. 2019;51(2):417-430.

2. Anderson B, LaPolla J, Turner D, Chapman G, Buller R. Ovarian transposition in cervical cancer. Gynecol Oncol. 1993;49(2): 206-214.

3. Buekers TE, Anderson B, Sorosky JI, Buller RE. Ovarian function after surgical treatment for cervical cancer. Gynecol Oncol. 2001;80(1):85-88.

4. Feeney DD, Moore DH, Look KY, Stehman FB, Sutton GP. The fate of the ovaries after radical hysterectomy and ovarian transposition. Gynecol Oncol. 1995;56(1):3-7.

5. Sonmezer M, Oktay K. Assisted reproduction and fertility preservation techniques in cancer patients. Curr Opin Endocrinol Diabetes Obes. 2008;15(6):514-522.

6. Haie-Meder C, Mlika-Cabanne N, Michel G, et al. Radiotherapy after ovarian transposition: ovarian function and fertility preservation. Int J Radiat Oncol Biol Phys. 1993;25(3):419-424.
7. Winarto H, Febia E, Purwoto G, Nuranna L. The need for laparoscopic ovarian transposition in young patients with cervical cancer undergoing radiotherapy. Int J Reprod Med. 2013;2013:1-6.

8. Absolom K, Eiser C, Turner L, et al. Ovarian failure following cancer treatment: current management and quality of life. Hum Reprod. 2008;23(11):2506-2512.

9. Falcone T, Attaran M, Bedaiwy MA, Goldberg JM. Ovarian function preservation in the cancer patient. Fertil Steril. 2004;81(2): 243-257.

10. Yoon A, Lee Y-Y, Park W, et al. Correlation between location of transposed ovary and function in cervical cancer patients who underwent radical hysterectomy. Int $J$ Gynecol Cancer. 2015;25(4):688-693.

11. Hoekman EJ, Knoester D, Peters AA, Jansen FW, de Kroon CD, Hilders CG. Ovarian survival after pelvic radiation: transposition until the age of 35 years. Arch Gynecol Obstet. 2018;298(5):1001-1007.

12. Hwang JH, Yoo HJ, Park SH, et al. Association between the location of transposed ovary and ovarian function in patients with uterine cervical cancer treated with (postoperative or primary) pelvic radiotherapy. Fertil Steril. 2012;97(6):1387-1393. e1382.

13. Swift BE, Leung E, Vicus D, Covens A. Laparoscopic ovarian transposition prior to pelvic radiation for gynecologic cancer. Gynecol Oncol Rep. 2018;24:78-82.

14. Damewood MD, Grochow LB. Prospects for fertility after chemotherapy or radiation for neoplastic disease. Fertil Steril. 1986;45(4):443459.

15. Wallace WH, Thomson AB, Saran F, Kelsey TW. Predicting age of ovarian failure after radiation to a field that includes the ovaries. Int J Radiat Oncol Biol Phys. 2005;62(3):738-744.

16. Howell SJ, Shalet SM. Fertility preservation and management of gonadal failure associated with lymphoma therapy. Curr Oncol Rep. 2002;4(5):443-452.

17. Du Z, Qu H. The relationship between ovarian function and ovarian limited dose in radiotherapy postoperation of ovarian transposition in young patients with cervical cancer. Cancer Med. 2017;6(3):508-515.

18. Morice P, Juncker L, Rey A, El-Hassan J, Haie-Meder C, Castaigne D. Ovarian transposition for patients with cervical carcinoma treated by radiosurgical combination. Fertil Steril. 2000;74(4):743-748.

19. Clough KB, Goffinet F, Labib A, et al. Laparoscopic unilateral ovarian transposition prior to irradiation: prospective study of 20 cases. Cancer. 1996;77(12):2638-2645.

20. Laios A, Duarte Portela S, Papadopoulou A, Gallos ID, Otify M, Ind T. Ovarian transposition and cervical cancer. Best Pract Res Clin Obstet Gynaecol. 2021;75:37-53.

21. Van Eijkeren MA, Van Der Wijk I, El Sharouni SY, Heintz AP. Benefits and side effects of lateral ovarian transposition (LOT) performed during radical hysterectomy and pelvic lymphadenectomy for early stage cervical cancer. Int $J$ Gynecol Cancer. 1999;9(5):396-400.

22. Faddy MJ, Gosden RG. A model conforming the decline in follicle numbers to the age of menopause in women. Hum Reprod. 1996;11(7):1484-1486.

23. Faddy MJ, Gosden RG, Gougeon A, Richardson SJ, Nelson JF. Accelerated disappearance of ovarian follicles in mid-life: 
implications for forecasting menopause. Hum Reprod. 1992;7(10):1342-1346.

24. Huang KG, Lee CL, Tsai CS, Han CM, Hwang LL. A new approach for laparoscopic ovarian transposition before pelvic irradiation. Gynecol Oncol. 2007;105(1):234-237.

25. Park YJ, Kim HS, Kang HC. The age at menopause and related factors in Korean women. J Korean Acad Nurs. 2002;32(7): 1024-1031.
26. Hoekman EJ, Broeders EA, Louwe LA, Nout RA, Jansen FW, de Kroon CD. Ovarian function after ovarian transposition and additional pelvic radiotherapy: a systematic review. Eur J Surg Oncol. 2019;45(8):1328-1340.

27. Chambers SK, Chambers JT, Holm C, Peschel RE, Schwartz PE. Sequelae of lateral ovarian transposition in unirradiated cervical cancer patients. Gynecol Oncol. 1990;39(2):155159. 